\title{
Safety Evaluation of Timber Structures through Probabilistic Analysis
}

\author{
Hélder S. Sousa ${ }^{1, a}$, Paulo B. Lourenço ${ }^{1, b}$ and Luís C. Neves ${ }^{2, c}$ \\ ${ }^{1}$ Universidade do Minho - Dept. of Civil Engineering, Azurém, 4800-085 Guimarães, Portugal \\ ${ }^{2} U N L$ - Universidade Nova de Lisboa, Quinta da Torre, 2829-516, Monte da Caparica, Portugal \\ ahssousa@civil.uminho.pt, ${ }^{b}$ pbl@civil.uminho.pt, ${ }^{9}$ lacn@fct.unl.pt
}

\begin{abstract}
This work presents a procedure for the use of probabilistic modelling techniques in the assessment of safety parameters of existing timber structures, when influenced by two types of actions. The parameters of the models have been defined as probabilistic variables, and Monte Carlo simulation technique was taken into account for safety evaluation. The case study here examined concerns four king post trusses on which a safety evaluation method was conducted to determine a target reliability index. Since the structures presented a high level of deterioration, a model considering the variation of the residual cross-section and the influence of environment in resistance was taken into account. Time dependent deterioration models have also been considered. With the results given by the different used models, it was possible to obtain probabilities of failure and respective reliability indices, as well as time evolution deterioration curves for a deteriorated historical structure.
\end{abstract}

Keywords: Timber trusses, reliability, remaining capacity factor, time evolution deterioration curves

\section{Introduction}

Timber, as a structural material, is present in many ancient buildings in Europe. However, some of these buildings present significant deterioration that limits their capability for use.

The purpose of this work is to apply a probabilistic methodology in a simplified way which, nevertheless, permits the analysis and assessment of ancient timber structures safety.

When dealing with existing structures, the use of semi-probabilistic methods, based on partial safety factors, is often over-conservative. Therefore the use of probabilistic methods is more appropriated in this case, since as the uncertainties in existing structures are quite different, and commonly smaller, than those present in structures to be built. Even more, since for existing structures a shorter remaining lifetime is expected, a lower safety margin may be considered. Bearing in mind these assumptions in a more detailed safety analysis, based on a probabilistic analysis, may take one to define that a structure is safe regardless of the fulfilment of the standard dimensioning codes.

Timber, as many natural materials, is susceptible to biological deterioration, being fungi and xylophagous insects its principal pathological agents. This decay phenomenon is more relevant in warmer climates, as those found in Southern Europe. These decay processes are visible by the destruction of the timber in contact with the surrounding atmosphere, which then progresses into the interior. The deterioration process in timber is different than the other materials, such as only a change in geometry is observed whereas the mechanical properties are not affected.

A thorough inspection of timber structures is often necessary because the deterioration of timber elements is heterogeneous along the structure, making possible to have both extremely damaged and very well preserved sections. The implementation of these data in spatial varying geometry, although recommended, is extremely complex and time consuming and for this reason, in this work, a set of simplified geometry models are proposed and compared, aiming to define a simple but accurate geometry modelling procedure for decayed timber structures. For this purpose, the safety of traditional timber structures is analysed over their entire life cycle by means of probabilistic analysis of the deterioration effect. A brittle failure of the timber structures was considered, in order to define the system reliability index. The geometric and mechanical properties of timber structures are considered as random variables, as are the loads and the effects of deterioration. 
The proposed methodology was applied to Chimico Laboratory's timber trusses since detailed inspections were available (Lourenço et al. 2005). This timber roof was recently demolished such as it presented a noteworthy deterioration due to biological attack, and it did not satisfy the codes in a semi-deterministic perspective.

\section{Case of study}

Chimico Laboratory's timber trusses The presented methodology was applied to the analysis of the timber trusses of the Chimico Laboratory, located in the University of Coimbra, Portugal. This building from the $18^{\text {th }}$ is an example of the Portuguese neoclassic, and was built as a research and teaching laboratory. It is composed of a three pitch roof and its initial structure had four king post timber trusses with $14.20 \mathrm{~m}$ of open span. During the requalification works for the construction of the Museum of Science, the trusses were removed considering their level of deterioration, due to xylophagous insects and wood-destroying fungi.

Structural modelling and loading A two-dimensional plane frame model was used for the discretization of the structure. Each timber element (tie beam, rafters, struts and post) was considered to have a linear axis and to have a hinge on both its end joints. Since a metal tie connected the lower ends of the principal rafters, the displacements on those points were restricted. Random variables were used for the definition of the mechanical properties of the materials and the applied loads. These random variables were defined by suitable probabilistic density functions, with adequate means and coefficients of variation according to non-destructive tests (Lourenço et al. 2005) and to probabilistic models (JCSS 2006). The value of the permanent load corresponded to the weight of the trusses, roof tiles and coating layer. Wind actions were calculated considering Eurocode 1.4 (CEN 2005) for both downwind and upwind mean exterior pressures. First and second order effects of loads in structural safety analysis were considered in the limit state functions (CEN 2004).

The information obtained by inspection with non-destructive methods carried out on the transversal sections (Lourenço et al. 2005), lead to a model for the residual cross-section area. For that purpose the area affected by xylophagous insects and wood-destroying fungi was considered to have a null resistance contribution. Also by this inspection it was found that two of the trusses were more degraded then the others. From now on, these trusses will be described as trusses 1 and 2, and the less degraded will be known as trusses 3 and 4 . Afterwards, four different approaches were considered in the modelling of the cross-sections dimensions. In the first three models, the cross-sections dimensions were modelled by deterministic values, such as: (i) the observed values at different sections; (ii) the mean of the observed values for each element and (iii) the minimum of the values observed for each element. The last model defined the cross-sections dimensions as random variables. Regarding the permanent load and wind load direction, three load combinations were considered, such as: (i) only permanent actions (Combination P); (ii) and (iii) both permanent and wind actions with consideration of wind direction (Combination PVI and Combination PV2). The value of $k_{\text {mod }}$ for Combination $P$ is 0.6, while for Combination PV1 and Combination PV2 the $k_{\text {mod }}$ value is 0.9 , considering the action with shortest duration (CEN 2004).

Deterministic cross-section models In order to assess the safety parameters of the first models, a Monte Carlo simulation method was applied considering random variables for the characteristics of the study case except for the cross-sections dimensions that were defined deterministically. Whenever high reliability indices were likely to be found, an approximation of the margin of safety by a normal distribution was used. Although only obtaining nominal probabilities of failure on those cases, this methodology was less time consuming. The results are presented in Table 1.

Cross-section defined by inspected values The cross-dimensions of the first model were obtained by the direct values obtained in the non-destructive inspection, such as the area attacked by insects and wood destroying fungi was not taken into account for the contribution of resistant area. The values obtained with this first model confirmed the lack of safety of trusses 1 and 2, whereas for this model and this type of decay mechanisms, trusses 3 and 4 still presented adequate performance. In all trusses, the limit state function that was more often violated corresponded to the buckling verification, since this model presented slender elements nearby the lower parts of the main rafters. 
However, this model may also be considered over-conservative such as it indicates that trusses 1 and 2 were extremely unsafe (see Figure 1, considering $\alpha=0$ ) when still standing while the non-destructive inspection was made, concluding that the decayed section area was still offering some resistance to cross-section specially when concerning the buckling limit state verification. The worst scenario was found when considering Combination $P$ because a smaller $k_{m o d}$ was used on that combination.

Cross-section defined by mean value in each element For this model it was assumed that there was a redistribution of stresses along each element and therefore localized small cross-sections were expected to have a less important influence in the safety assessment. By considering the mean value of the dimensions of the cross-sections for each element it was found, as one could expect, that the elimination of those localized small cross-section increased the reliability indices. Nevertheless, comparing the four trusses it was found that the overall mechanical behaviour was equivalent to the previous model, such as trusses 1 and 2 were still the ones with higher risk of collapse.

Cross-section defined by minimum value in each element When dealing with the possibility of a brittle failure, the probability of collapse occurring due to the most degraded sections is very high, and therefore the consideration of the minimum value in each element for the cross-section is plausible. For this model, the probabilities of failure for trusses 1 and 2 highly increase characterizing these trusses as extremely unsafe and also defining truss 4 as unsafe. This model is over-conservative when studying truss 1 , because it states that this truss will must certainly fall, situation which was not found in situ. Truss 3 still offers acceptable reliability indices because its degradation did not lead to small cross-sections.

Table 1: Reliability indices for the different deterministic cross-section models

\begin{tabular}{cccccccccc}
\hline Truss & \multicolumn{4}{c}{ Inspected values } & \multicolumn{4}{c}{ Mean value by element } & \multicolumn{3}{c}{ Minimum value by element } \\
& $\mathrm{P}$ & $\mathrm{PV} 1$ & $\mathrm{PV} 2$ & $\mathrm{P}$ & $\mathrm{PV} 1$ & $\mathrm{PV} 2$ & $\mathrm{P}$ & $\mathrm{PV} 1$ & $\mathrm{PV} 2$ \\
\hline 1 & -1.374 & 0.905 & -0.356 & 2.208 & 3.771 & 3.341 & $-\mathrm{Inf}$ & $-\mathrm{Inf}$ & $-\mathrm{Inf}$ \\
2 & -2.435 & -1.231 & 0.140 & 1.171 & 2.238 & 3.504 & -2.578 & -1.348 & -0.279 \\
3 & 3.917 & 5.962 & 5.039 & 5.186 & 6.509 & 6.032 & 3.284 & 5.091 & 4.341 \\
4 & 4.184 & 4.942 & 7.066 & 3.985 & 4.768 & 5.605 & -2.808 & -1.200 & -0.291 \\
\hline
\end{tabular}

Residual cross-section variation The assumption that the decayed area had a null resistance leads to over-conservative values. Therefore, a model where the contribution of the decayed area to the global resistance of the residual cross-section was found to be necessary especially for the most decayed trusses (trusses 1 and 2). For this purpose, a remaining capacity factor, $\alpha$, was considered, such that the final residual cross-section area would be defined as in Eq. 1.

$$
A_{f}=A_{N D}+\alpha \cdot A_{D}
$$

where $A_{f}$ is considered to be the residual cross-section for each element, $A_{N D}$ is the area which was not attacked by neither xylophagous insects nor wood-destroying fungi, $A_{D}$ is the area which was attacked by any kind of decay agent and $\alpha$ is the remaining capacity factor. The considered model corresponded to the deterministic cross-section model with inspected values for the cross-sections. The evolution of the reliability index in terms of the remaining capacity factor is shown for truss 1 , as an example in Figure 1, where a maximum limit of 50\% was considered for $\alpha$. 


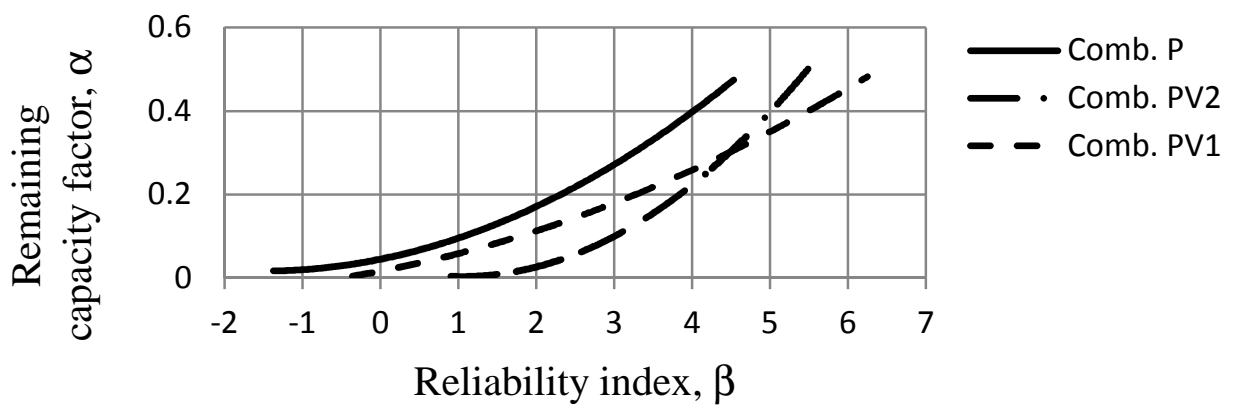

Figure 1: Evolution of $\beta$ with remaining capacity factor, $\alpha$ : truss 1

From this analysis it is concluded that the consideration of a coefficient $\alpha$ is more important as the value of $A_{D}$ is higher. Therefore, trusses 1 and 2, which are more decayed, have a higher increment in their reliability indices than trusses 3 and 4. Taking a value close to 0 for target reliability index, since the trusses were still standing despite their level of deterioration, it was found that a $25 \%$ value of $\alpha$ for the most decayed truss would be suitable for safety evaluation of that structure.

Probabilistic cross-section model To consider a probabilistic cross-section model a lognormal distribution with mean value given by the mean of the observed values and a coefficient of variation of $10 \%$ was taken into consideration. This model led to a decrease in the overall reliability indices mainly due to the consideration of a new random variable, which increases the level of uncertainty of the structural characterization. Nevertheless, the consideration of a full probabilistic parameter to define the geometry of an existing timber structure is more suitable because the mechanical properties of timber vary along the different elements and also it avoids a time consuming inspection. When studying the most decayed truss, the obtained reliability indices are equivalent to those found in the residual cross-section variation model with a $25 \%$ remaining capacity factor.

Variation of the parameter $\boldsymbol{k}_{\text {mod }}$ The Eurocode 5 (CEN 2004) analysis defines a $k_{\text {mod }}$ factor depending on three different service classes regarding the type of timber, use and surrounding environmental conditions. When a load combination has different types of loads, this code also recommends the use of the $k_{\text {mod }}$ factor concerning the load with smaller duration. Since this factor influences both the design values for mechanical and resistant properties of timber, its contribution is very important in safety analysis of a timber structure. In this case, the consideration of different $k_{\bmod }$ factors for the different types of load combinations led to the situation that the increment of a new variable load would increase the reliability indices, which for decayed structures may pose a physical inconsistency. Therefore, to better characterize this case study a set of simulations was made in order to find a more suitable methodology to implement the $k_{\text {mod }}$ factor.

Linear variation of $\boldsymbol{k}_{\text {mod }}$ factor Firstly the reliability indices for the most decayed truss (truss 2) were calculated varying linearly the $k_{\text {mod }}$ factor between 0.6 and 0.9 for all load combinations. The results are shown in Figure 2 for both the deterministic and probabilistic cross-section models.

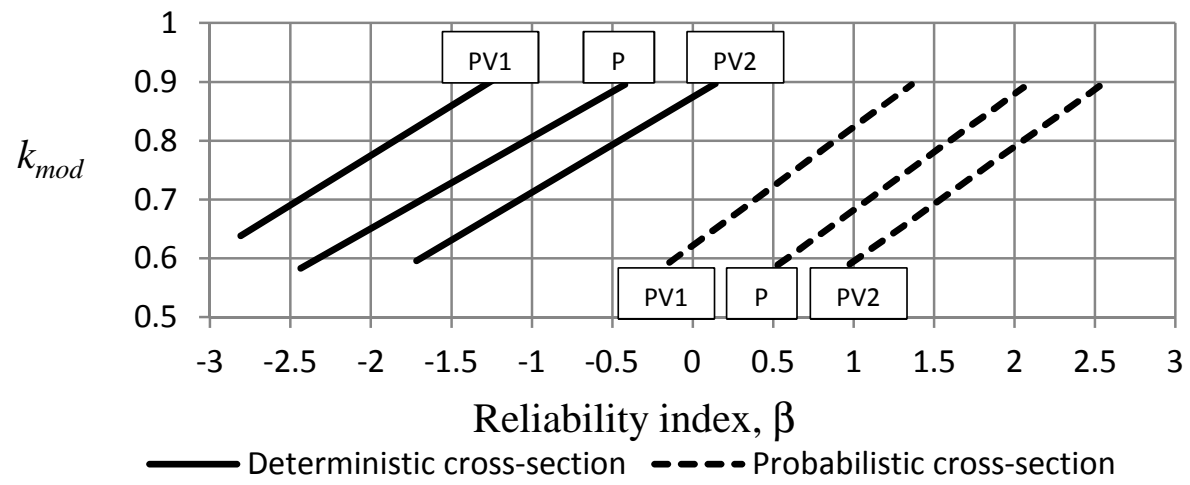

Figure 2: Linear variation of $k_{\text {mod }}$ factor: truss 2 
The previous figure shows that the evolution of the reliability indices is linear with the variation of the $k_{\text {mod }}$ factor. For all the combinations, in both deterministic and probabilistic models, the tendency line has similar inclinations.

Different $\boldsymbol{k}_{\text {mod }}$ factors for each separate load A different approach to find a suitable methodology for the attribution of the $k_{\text {mod }}$ factor was to give separate values of this parameter to the several different loads and then implement that in the calculus of the limit state function and related safety margin, as:

$$
M=1-\sum_{i=1}^{n}\left(R_{1} / S_{1}+R_{2} / S_{2}+\ldots+R_{n} / S_{n}\right)
$$

where each $R_{i}$ and $S_{i}$ corresponds respectively to the resistance and to the load effects of a determine load type with the associate $k_{\text {mod }}$ factor.

The smaller reliability indices obtained by this procedure are now found for the load combinations with both permanent and wind actions, which are the load combinations that produce a higher level of stresses in the structures.

Time evolution deterioration curves To assess the evolution of the timber elements deterioration a bi-parametrical idealized decay model (Leicester 2001) was considered. The two parameters correspond to an initial propagation period of the deterioration phenomenon, $t_{\text {lag }}$ (year) and an annual penetration ratio, $r(\mathrm{~mm} / \mathrm{year})$. Considering a probabilistic analysis, the $t_{\text {lag }}$ parameter is to be defined as a deterministic variable, while the $r$ parameter should be defined by a lognormal distribution with a coefficient of variation between 0.5 and 1 according to the timber durability class (Wang et al. 2006). These parameters were obtained by an iterative process, by adjusting the reliability indices attained for each set of the model's parameters with the reliability indices calculated in the previous mentioned geometric models. This procedure resulted in having different values of $r$ depending on the end of lifetime reliability indices for each load combination and model: (i) deterministic cross-section, inspected values; (ii) residual cross-section variation, $\alpha=25 \%$; (iii) probabilistic cross-section. After computing these parameters it was possible to represent time evolution deterioration curves for the most decayed truss. As an example the results obtained considering a deterministic cross-section defined in terms of the values observed in the inspection is presented in Figure 3.

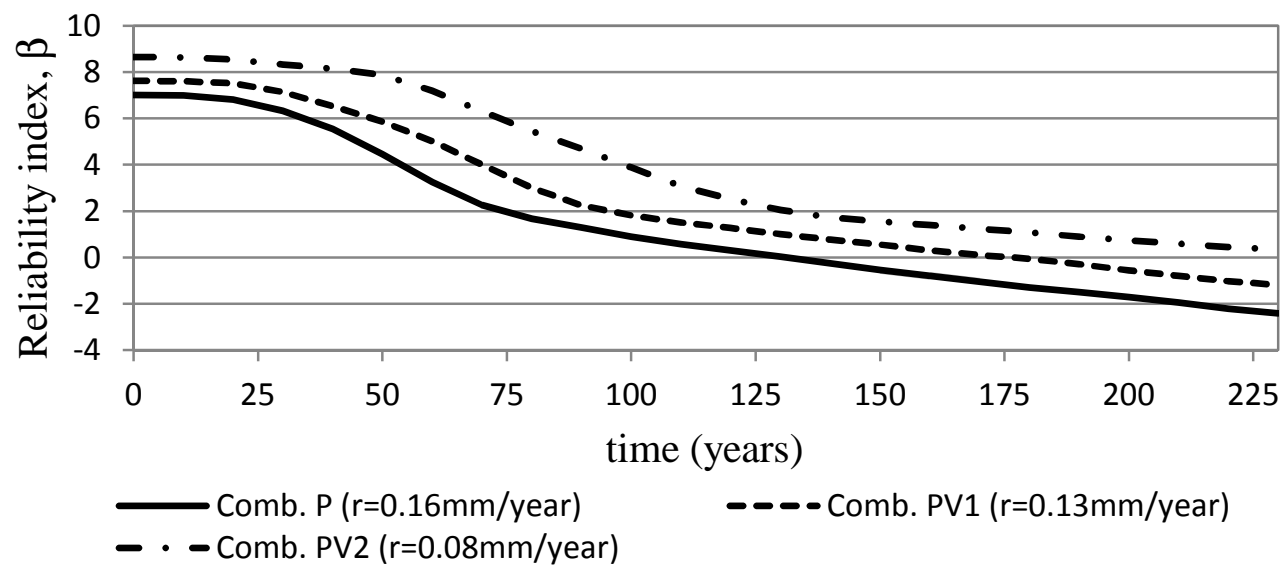

Figure 3: Time evolution deterioration curves for truss 2 and deterministic cross-section model with inspected values

The time evolution deterioration curves on the deterministic cross-sections on all the different load combinations have similar derivatives. The principal difference between then is a vertical translation of the curves. All models distinguish three different phases on the evolution of the reliability index along time. In the early life of the structure, while the influence of the parameter $t_{\text {lag }}$ is still present, high reliability indices are found and are kept almost constant. After that phase, a significant decrease in the reliability indices is presented since the decay phenomenon is now fully active. Finally, the last 
phase presents a less significant decrease of the reliability indices because the structure is already very much damaged. For the two other models the Combination PV2 has a slightly different behaviour compared with the other load combinations. Since the $k_{\text {mod }}$ factor was kept in accordance to the Eurocode 5 (CEN, 2004) analysis the curves for Combination $P$ always present the lower reliability indices.

\section{Conclusions}

The presented work described a possible simplified procedure to evaluate the safety level of existing timber structures, in a probabilistic point of view. This methodology also provides guidance on how to define a maintenance and preservation plan, by predicting a possible behaviour of the deterioration phenomenon, since suitable modelling methods are taken into consideration.

The importance of defining a suitable geometrical model for safety assessment of decayed timber structures was also emphasized in this work and an adequate target reliability index was assumed for that purpose. When studying the contribution of the decayed areas for global resistance in the most decayed truss, a remaining capacity factor of approximately $25 \%$ was found to be suitable. Similar safety parameters were found in a probabilistic cross-section model with a $10 \%$ coefficient of variation.

To better analyse the influence of the $k_{\text {mod }}$ factor two different methods were applied, from which a consideration of different $k_{\text {mod }}$ factors for each separate load provided more adequate results for the case of the timber roof structure of Chimico Laboratory.

An idealized decay model with time evolution deterioration curves was also taken into consideration, making possible to determine how a maintenance plan should have been implemented in order to increase the lifetime of the structure.

Despite the work here presented, there is still room for geometric modelling improvement by taking into account local damage, such as nodes and cracking of the wood, on the probabilistic models.

\section{Acknowledgments}

The financial support of the Portuguese Science Foundation (Fundação de Ciência e Tecnologia, FCT), through project PTDC/66527-2006, is gratefully acknowledged. The third author acknowledges the support of FCT through research centre UNIC

\section{References}

[1] Lourenço, P. B., Valle, A. and Brites, R.D. (2005). Levantamento do estado de degradação das Asnas da Cobertura do Laboratório Chimico - Al, Coimbra, Civil Engineering Department of the University of Minho, Guimarães, Portugal (in Portuguese).

[2] Joint Committee on Structural Safety (2006). JCSS Probabilistic Model Code, Part 3: Resistance Models - 3.5 Properties of Timber. Probabilistic Model Code, Available: www.jcss.ethz.ch.

[3] CEN (2005). ENV 1991-1-4 - Eurocode 1 - Actions on structures - Part 1-4: General actions Wind actions, European Committee for Standardization (CEN), Brussels.

[4] CEN (2004). ENV 1995-1-1 - Eurocode 5 - Design of Timber Structures - Part 1-1: General Common rules and rules for buildings, European Committee for Standardization (CEN), Brussels.

[5] Leicester, R.H. (2001). Engineered durability for timber construction, John Wiley \& Sons, Ltd., CSIRO, Highett, Australia, pp 216-227.

[6] Wang, C, Leicester, R.H. and Nguyen, M. (2006). Probabilistic procedure for design of untreated timber poles in-ground under attack of decay fungi, CSIRO Division of Sustainable Ecosystems, Australia, Available: www.sciencedirect.com. 